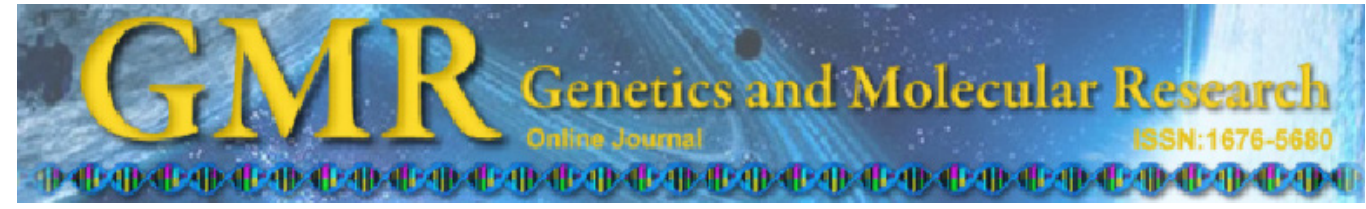

\title{
Promoter methylation negatively correlated with mRNA expression but not tissue differential expression after heat stress
}

\author{
J.K. Gan ${ }^{1,2}$,D.X. Zhang ${ }^{1,2}$, D.L. He ${ }^{2,3}$, X.Q. Zhang ${ }^{1,2}$, Z.Y. Chen ${ }^{1,2}$ and Q.B. Luo ${ }^{1,2}$ \\ ${ }^{1}$ Department of Animal Genetics, Breeding and Reproduction, \\ College of Animal Science, South China Agricultural University, \\ Guangzhou, Guangdong, China \\ ${ }^{2}$ Guangdong Provincial Key Lab of Agro-Animal Genomics and Molecular \\ Breeding, Guangzhou, Guangdong, China \\ ${ }^{3}$ Key Lab of Chicken Genetics, Breeding and Reproduction, \\ Ministry of Agriculture, South China Agricultural University, Guangzhou, China \\ Corresponding author: Q.B. Luo \\ E-mail: qbluo@scau.edu.cn \\ Genet. Mol. Res. 12 (1): 809-819 (2013) \\ Received January 10, 2012 \\ Accepted November 18, 2012 \\ Published March 15, 2013 \\ DOI http://dx.doi.org/10.4238/2013.March.15.1
}

\begin{abstract}
DNA methylation plays a central role in gene expression. In this study, we detected the promoter methylation pattern of the chicken heat shock protein 70 (HSP 70) gene and its association with messenger RNA (mRNA) expression before and after heat shock. The results showed that mRNA expression increased in response to heat stress and peaked at $3 \mathrm{~h}$ before dropping. Hypomethylation of the HSP 70 promoter occurred in all of the groups studied, but the difference between groups within tissue type was not significant. The DNA methylation level of the control and the 6-h treatment groups was slightly higher than that of the 3-h treatment group in brain tissue and leg muscle. Correlation analysis between mRNA expression and DNA methylation of HSP70 showed that DNA methylation was negatively associated with mRNA expression in leg muscle $(\mathrm{P}=0.0124)$, indicating that DNA methylation may be negatively associated with the expression of $H S P 70$, although the difference was not significant. We concluded that the expression of HSP70 is heat inducible and tissue dependent and that heat induction
\end{abstract}


may correlate with DNA methylation pattern in the HSP70 promoter, whereas tissue dependence is unrelated to DNA methylation pattern.

Key words: HSP70; DNA methylation; Heat inducible

\section{INTRODUCTION}

Heat shock protein 70 (HSP70) is a member of the heat shock protein family. As a molecular chaperone, it is required for the correct folding of newly synthesized proteins and the degradation of misfolded proteins (Hartl, 1996; Mayer and Bukau, 2005). HSP70 is also involved in apoptosis inhibition and cell cycle regulation (Kregel, 2002). More important, HSP70 plays a critical role in the regulation of the heat shock response and the acquisition of thermotolerance (Mayer and Bukau, 2005). In view of these important roles, HSP70 is a proposed biomarker for the study of environmental stress in chickens (Maak et al., 2003).

The expression of HSP70 has been demonstrated to be heat inducible and occur rapidly after transient heat stress (Wang and Edens, 1998), but the expression of HSP70 differs according to individuals and tissue type. Compared with normally feathered birds, heat-resistance birds (naked-neck Label Rouge) display lower HSP70 levels in hepatic cells when gradual heat stress is induced (Mazzi et al., 2002). Guerreiro et al. (2004) measured brain and hepatic HSP70 protein levels using Western blot after gradual heat stress and found that brain HSP70 levels are 3-4 times higher than those in the hepatic tissue of heat-stressed birds reared at thermoneutrality. Much research has been performed to illuminate the cause of HSP70 expression differences in individuals and tissue-dependent expression. Mahmoud (2000) has analyzed HSP7O in birds submitted to heat stress and found that more resistant birds have only 1 Pst I HSP70 allele upstream from the coding region, whereas the other breeds had 2 different alleles for that gene. Zhang et al. (2002) also detected polymorphism in regulatory and coding regions of $H S P 70$ in chickens with various heat tolerance capabilities using polymerase chain reaction (PCR) single-strand conformation polymorphism analysis and sequencing. Mazzi et al. (2003) analyzed the promoter region and the beginning of the coding region of $H S P 70$ in chickens with various heat tolerance capabilities and found 2 single nucleotide polymorphisms (SNPs) - A+258G and C+276G - both of which were silent mutations. Zhen et al. (2006) tested the chicken HSP70 mRNA expression of various genotypes at polymorphism sites $\mathrm{C}+276 \mathrm{G}$ and $\mathrm{A}+258 \mathrm{G}$ in liver and leg muscle tissue using real-time quantitative PCR (qPCR). The results showed that the heterozygote has a higher expression level than that of other genotypes at these polymorphism sites.

These studies are illuminative but still fail to illustrate why HSP70 is heat inducible and why its expression is tissue dependent, because SNPs in the chicken HSP70 identified to date are not localized to the heat shock element (HSE), CAAT box, SP1 binding sites, TATAA box, or any other cis-elements in the regulatory region, and they have not been found to affect the binding activity of heat-shock transcription factors (HSFs). Therefore, the aim of the present study was to determine the cause of $H S P 70$ differential expression from another angle - epigenetics. DNA methylation is an epigenetic modification that plays an important role in the modulation of gene expression. Aberrant DNA methylation is associated with gene silencing or downregulation (Bird, 2002; Dai et al., 2003). Studies of human diseases have revealed that complex diseases such as cancer are often associated with sudden hypermethylation of 
tumor suppressor genes or hypomethylation of cancer-related genes (Robertson and Wolffe, 2000; Brena et al., 2006). Methylation of the CpG island within the promoter prevents protein binding at DNA methylation sites, directly inhibiting gene transcription because when double-stranded DNA forms a three-dimensional structure, the methyl of methylated cytosine may be highlighted in the major groove, blocking the interaction between transcription factors and genes (Kuroda et al., 2009). Normally the gene CpG island is unmethylated (Straussman et al., 2009). Xu et al. (2007) measured the genome-wide DNA methylation level of 3 chicken strains, and the average incidence of DNA methylation was approximately $29.4 \%$ in muscle, $27.2 \%$ in liver, $27.2 \%$ in heart, and $26.1 \%$ in kidney, indicating that the methylation level of the chicken genome is low. However, when the organism is subjected to certain stimuli, the methylation level may change to a certain extent. Xing et al. (2011) fed betaine to broilers and found that mRNA expression of the lipogenesis gene decreased significantly, possibly owing to the upregulation of DNA methylation in some $\mathrm{CpG}$ sites within the lipogenesis gene promoter. Yossifoff et al. (2008) found that transferred 3-day-old birds to $37.5^{\circ} \mathrm{C}$ for $24 \mathrm{~h}$ caused transient changes in the expression of brain-derived neurotrophic factor that coincided with changes in the $\mathrm{CpG}$ methylation pattern in the avian brain-derived neurotrophic factor promoter region. Our preliminary research has uncovered a $\mathrm{CpG}$ island with high GC content in the 5'-flanking region of HSP70. DNA methylation may occur more easily in regions with high GC content (Watanabe and Maekawa, 2010).

Given the results of previous studies and our preliminary findings, we hypothesized that DNA methylation may play a role in the heat-inducible and tissue-dependent expression of chicken HSP70. To verify the hypothesis, we subjected 8-day-old female chicks to acute heat stress $\left(40^{\circ} \mathrm{C}\right)$, and liver, brain, and leg muscle tissue were sampled for RNA and DNA preparation at various time points. The $H S P 70$ mRNA and DNA methylation levels of the tissues were analyzed, and correlations between mRNA expression and DNA methylation were explored to determine whether the methylation pattern of the HSP70 promoter is associated with the differential expression of $H S P 70$.

\section{MATERIAL AND METHODS}

\section{Chicks and experimental design}

Eighty female chicks (Dwarf Yellow Chicken) were obtained from the Guangdong Wens South China Poultry Breeding Company (Guangdong, China) on the day of hatching and raised in an adequate environmental temperature under continuous artificial illumination and ad libitum access to food and water. At 8 days of age, 64 chicks were transferred to an artificial climate chamber (LH-100-RDCT, Japan) in which the temperature was $40^{\circ} \mathrm{C}$ and the humidity was $80 \%$ for $6 \mathrm{~h}$. The remaining 16 chicks served as controls and were left in a thermoneutrality room (ambient temperature, $30^{\circ} \mathrm{C}$ ). After heat stress for 1, 2, 3, and $6 \mathrm{~h}, 16$ chicks were caught randomly for peripheral blood and sample collections.

\section{Tissue collection, RNA, and DNA preparation}

Peripheral blood from all of the chicks was collected from the heart, and the chicks were killed via cervical dislocation. Liver, brain, and leg muscle tissue were sampled for DNA 
and RNA preparation and immediately placed in liquid nitrogen. Tissue RNA was extracted using the TRI reagent (TaKaRa, Japan) and tissue DNA was extracted using a TaKaRa Universal Genomic DNA Extraction Kit 3.0 (TaKaRa) according to manufacturer instructions. Genomic DNA was extracted from blood using the phenol-chloroform method.

\section{Real-time qPCR}

First-strand complementary DNA was synthesized from total RNA using a ReverTra Ace qPCR RT Kit (TOYOBO, Japan). Samples were then analyzed with real-time qPCR using SYBR Green Real-Time PCR Master Mix (TOYOBO). Real-time qPCRs were performed in a final volume of $20 \mu \mathrm{L}$ according to manufacturer instructions. Expression was determined by comparing the $H S P 70$ mRNA levels with those of $\beta$-actin, and the final expression level was standardized through comparison to the expression level of the liver tissue in the control group. The following primers were used for real-time qPCR: HSP70: F: 5'-GCGCCAGGCC ACCAAAGATG-3'; R: 5'-GCCCCCTCCCAAGTCAAAGATG-3'; $\beta$-actin: F: 5'-CTCCCCCA TGCCATCCTCCGTCTG-3'; R: 5'-GCTGTGGCCATCTCCTGCTC-3'.

\section{Amplification of the HSP70 promoter region}

The promoter region of a gene is normally positioned between $2500 \mathrm{bp}$ upstream and $200 \mathrm{bp}$ downstream of nucleotide $\mathrm{A}$ in the initiator consensus sequence. The promoter region sequence was downloaded using the EBI Ensembl Tool (http://www.ensembl.org/index.html). Primers designed to amplify the promoter region were as follows: F: 5'-AATCGACACCCTT CCCATCCA-3'; R: 5'-TGGTGTTGGTGGGGTTCATTG-3'. The amplification product was $2020 \mathrm{bp}$.

\section{Analysis of the HSP70 promoter region CpG islands}

The Online software (http://www.urogene.org/methprimer/index1.html) was used to analyze the $\mathrm{CpG}$ islands in the promoter region of $H S P 70$.

\section{DNA methylation assay of the CpG island}

Quantitative high-throughput DNA methylation analysis was performed using the MassARRAY system as described elsewhere (Ehrich et al., 2005). Two bisulfite reactions (A1 and A2) for detecting the methylation level of the CpG island in the HSP70 promoter were designed, covering 31 and 39 CpGs. The following primers were used for the reactions: A1, F: 5'-ACGTTTTTGATTGGGTAGGAGGTAA-3'; R: 5'-AACAATAATCTCCACTTTACCA TAC-3', covering a region of $365 \mathrm{bp}$. A2, F: 5'-ATTTTTTATTTTGAGGGAAAGGGGT-3'; R: 5'-ACCTCCTACCCAATCAAAAACGT-3', covering a region of $496 \mathrm{bp}$. The methylation levels of the control, 3-, and 6-h treatment groups were analyzed.

\section{Expression vectors and luciferase reporter assay}

Because the CpGs in the HSP70 gene promoter are too numerous for us to study, we 
focused on finding the core promoter of HSP70 and analyzing the CpGs within it. A PGL3 basic vector (Promega, USA) was double-restriction enzyme digested and used to ligate the truncation amplification products of the $H S P 70$ promoter to determine the core promoter. Seven expression vectors called $1 \mathrm{~F}, 2 \mathrm{~F}, 3 \mathrm{~F}, 4 \mathrm{~F}, 5 \mathrm{~F}, 6 \mathrm{~F}$, and $7 \mathrm{~F}$ were constructed; the primers used to amplify the HSP70 promoter are shown in Table 1. The PGL3 basic vector served as a negative control, and the PGL3 promoter vector served as a positive control. The PCR products were ligated into the pGL3 basic vector between the MluI and XhoI site. The plasmids were then transfected into chicken embryo fibroblasts at a density of $1.5 \times 10^{5}$ cells/well. A 24-well plate was used with $500 \mu \mathrm{L}$ medium containing 5\% serum for each well. A total of $1 \mu \mathrm{g}$ plasmid pGL3 vector and $500 \mathrm{ng}$ pRL-TK internal control vector (Promega) were cotransfected into cells using Lipofectamine ${ }^{\mathrm{TM}} 2000$ (Invitrogen, USA). After 6 h, the medium was changed to fresh medium with $10 \%$ fetal bovine serum. The cells were then incubated for $48 \mathrm{~h}$, and the luciferase assay was performed according to manufacturer instructions (Promega). Luciferase activity was normalized using pRL-TK activity. Each experiment was performed 6 times.

Table 1. List of primers used in the truncation amplification of the promoter region of HSP70.

\begin{tabular}{ll}
\hline Primer & Sequences $\left(5^{\prime} \rightarrow 3^{\prime}\right)$ \\
\hline P1F & $g c g$ ACGCGTATTTCTCCAAGGTCAA \\
P2F & $g c g$ ACGCGTGCTGCACTTTTAACAT \\
P3F & gcgACGCGTTTAGCACAACAGACAA \\
P4F & ataACGCGTCTGGCATAGAGGGAGC \\
P5F & ataACGCGTCCCCCGAGAGGCAGTG \\
P6F & ataACGCGTGAGGGTTGGGCTAGAG \\
P7F & act $\underline{\text { ACGCGTGAGGAATCTATCATCA }}$ \\
PR & atCGAGGTGTTGGTGGGGTTCA \\
\hline
\end{tabular}

Nucleotides in lower case letters are protective bases; underlined bases are restriction enzyme recognition sequences: ACGCGT is recognized by $M l u \mathrm{I}$ and CTCGAG is recognized by XhoI.

\section{Statistical analysis}

The relative expression levels of $H S P 70$ were determined with $2^{-\Delta \Delta \mathrm{Ct}}$, for which $\Delta \mathrm{Ct}$ is $\mathrm{Ct}_{\text {target gene }}$ minus $\mathrm{Ct}_{\beta \text {-actin }}$ and $\Delta \Delta \mathrm{Ct}$ is $\Delta \mathrm{Ct}$ minus $\Delta \mathrm{Ct}_{\text {standard value }}$. Differences between the groups in all the biochemical studies were examined using SAS 8.2 with a general linear model procedure. Correlation analysis between methylation and mRNA expression levels of $H S P 70$ was carried out using SAS 8.2 with a CORR procedure. A value of $\mathrm{P}<0.05$ was considered to be statistically significant.

\section{RESULTS}

\section{HSP70 mRNA expression}

Real-time qPCR analysis revealed that compared to expression in control tissues, HSP70 mRNA expression increased in response to heat stress and peaked at $3 \mathrm{~h}$ at levels 40 times higher in liver tissue, 19 times higher in brain, and 2.8 times higher in leg muscle, then 
dropped at $6 \mathrm{~h}$ (Figure $1 \mathrm{~A}-\mathrm{C}$ ). The expression levels in liver and brain in chicks treated for $3 \mathrm{~h}$ were significantly different than those in other groups $(\mathrm{P}<0.01)$, whereas the expression levels in leg muscle in chicks treated for $3 \mathrm{~h}$ were significantly different than those of other groups $(\mathrm{P}<0.01)$ with the exception of the 6-h treatment group $(\mathrm{P}=0.08)$. HSP70 expression was higher in leg muscle, followed by brain and liver tissue.
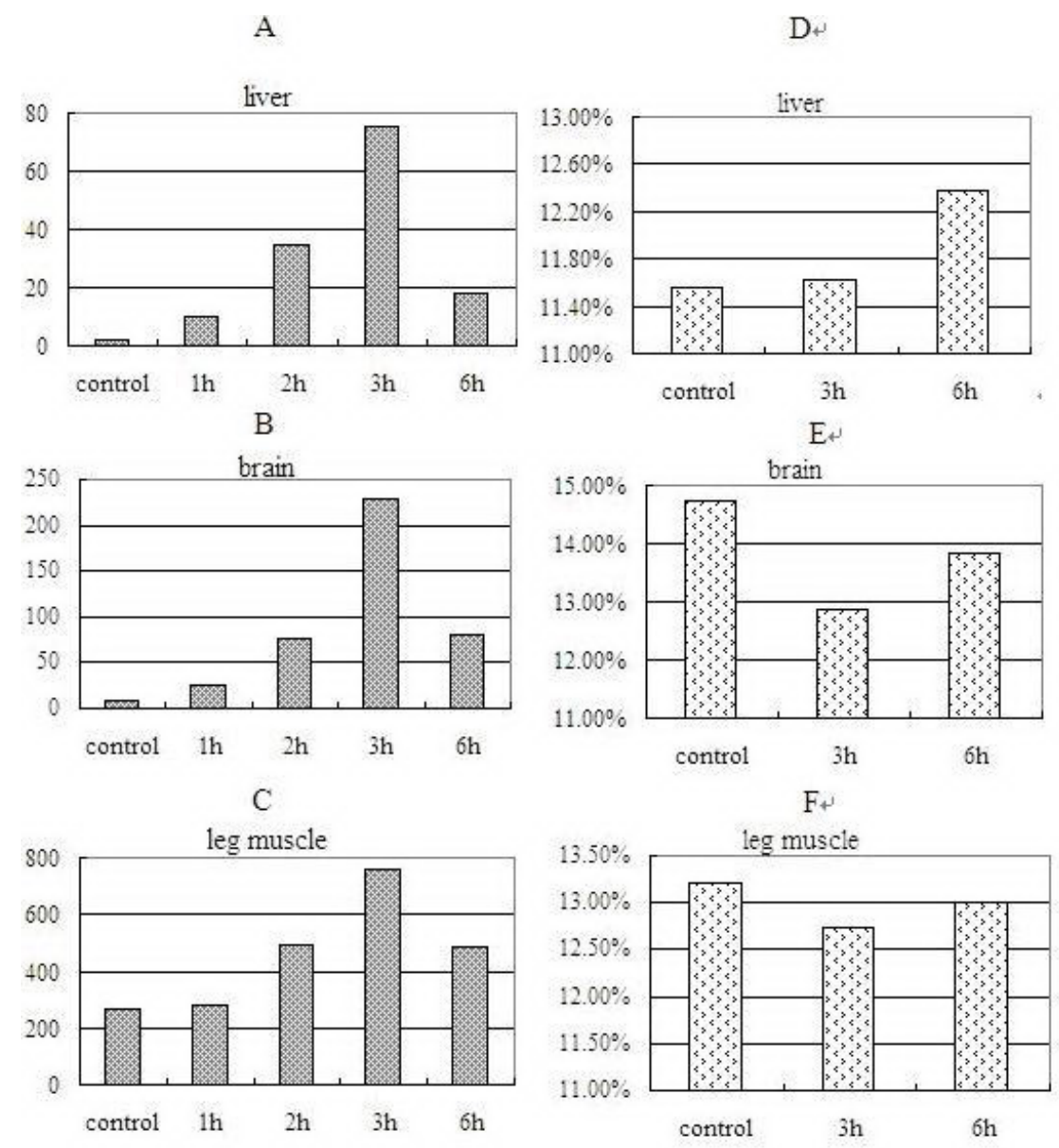

Figure 1. A. B. C. mRNA expression level of $H S P 70$ in different tissues at different times. D. E. F. Promoter methylation level of HSP70 in different tissues at control, 3 and $6 \mathrm{~h}$.

\section{DNA methylation of the HSP70 promoter CpG island}

The CpG island region of the HSP70 promoter was 1068 bp according to the Online software analysis (see Figure 2). The total average methylation incidences of the control, 3-, and 6-h treatment groups were $11.56 \pm 1.42,11.62 \pm 1.59$, and $12.38 \pm 2.08 \%$, respectively, in liver tissue (see Figure 1D); $14.73 \pm 1.68,12.87 \pm 1.33$, and $13.82 \pm 2.05 \%$, respectively, in brain tissue (see Figure 1E), and 13.22 $\pm 4.77,12.74 \pm 2.46$, and $13.03 \pm 1.69 \%$, respectively, 
in leg muscle (see Figure 1F). The differences within tissue groups were not significant $(\mathrm{P}>$ $0.05)$, and correlation analysis showed that methylation level was negatively associated with mRNA expression in leg muscle $(\mathrm{P}=0.0124)$.

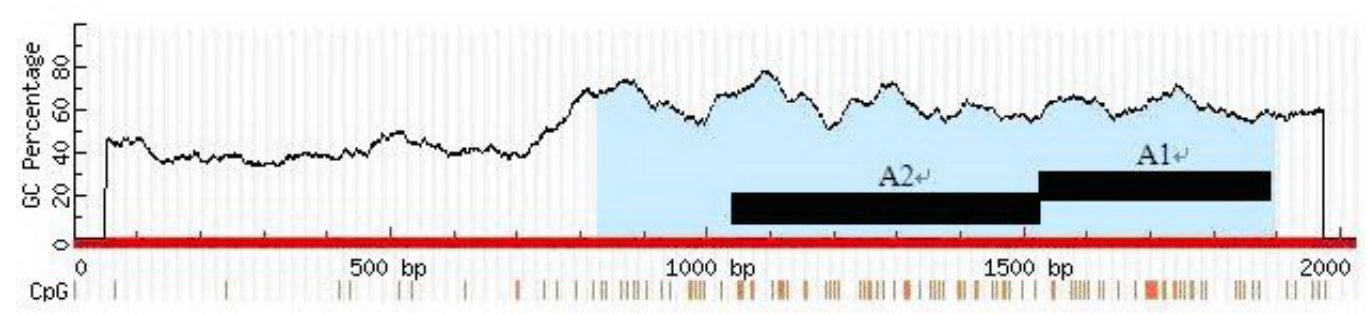

Figure 2. $\mathrm{CpG}$ island of $H S P 70$ promoter. The light blue region is the $\mathrm{CpG}$ island of $H S P 70$, the black thick lines marked by $\mathrm{A} 1$ and $\mathrm{A} 2$ are the methylation detection regions.

\section{Luciferase reporter assay of core promoter activity}

The dual-luciferase reporter assay showed similar values in vectors $5 \mathrm{~F}$ and $6 \mathrm{~F}$, with slightly higher values in $5 \mathrm{~F}$, which had higher values than any other expression vectors, suggesting that compared with $5 \mathrm{~F}$, the missing fragment of $7 \mathrm{~F}$ could be the core promoter (-546 to $-15 \mathrm{bp})$. The expression value of $2 \mathrm{~F}$ was higher than that of $1 \mathrm{~F}$, suggesting that compared with $1 \mathrm{~F}$, the missing fragment of $2 \mathrm{~F}$ may contain transcription factors that inhibit the transcription of $H S P 70$ (Figure 3).

ש expression level

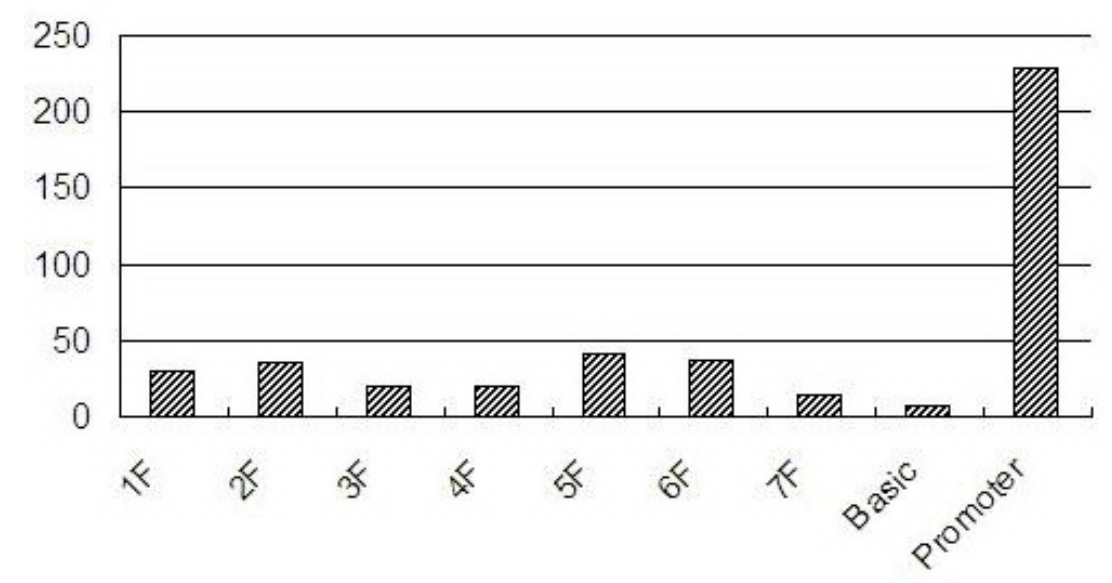

Figure 3. Dual-luciferase reporter assay result. 1F, 2F, 3F, 4F, 5F, 6F, and 7F represent different expression vectors. Basic is the negative control and promoter is the positive control. 


\section{Methylation levels of core promoter transcription sites}

The transcription factors of the $H S P 70$ core promoter were analyzed using the Online software (http://www.genomatix.de/en/index.html). As shown in Figure 4, the CpG sites at positions $-426,-419$, and -413 formed a $\mathrm{CpG}$ cluster. The analysis revealed that this region was an SP1 transcription factor, and the methylation levels of this region in the control, 3-, and 6-h treatment groups were $25.00 \pm 3.89,22.88 \pm 2.95$, and $26.25 \pm 3.49 \%$, respectively, in liver tissue; $27.43 \pm 3.60,25.50 \pm 5.73$, and $23.29 \pm 4.07 \%$, respectively, in brain tissue, and 25.86 $\pm 6.72,24.25 \pm 3.20$, and $26.25 \pm 2.20 \%$, respectively, in leg muscle. None of the differences within tissue groups was significant $(\mathrm{P}>0.05)$. The $\mathrm{CpG}$ sites at positions $-243,-239$, and -232 formed another $\mathrm{CpG}$ cluster. Online software analysis showed that this region was also an SP1 transcription factor, but the methylation levels of this region in the control, 3-, and 6-h treatment groups showed no consensus with those above (data not shown). In addition, a CpG site at position -172 in the HSE, which is the HSF binding site, displayed hypomethylation at a level $6 \%$ lower than that in any of the groups (data not shown). Correlation between the DNA methylation of the transcription factor CpGs and HSP70 expression was not significant (data not shown).

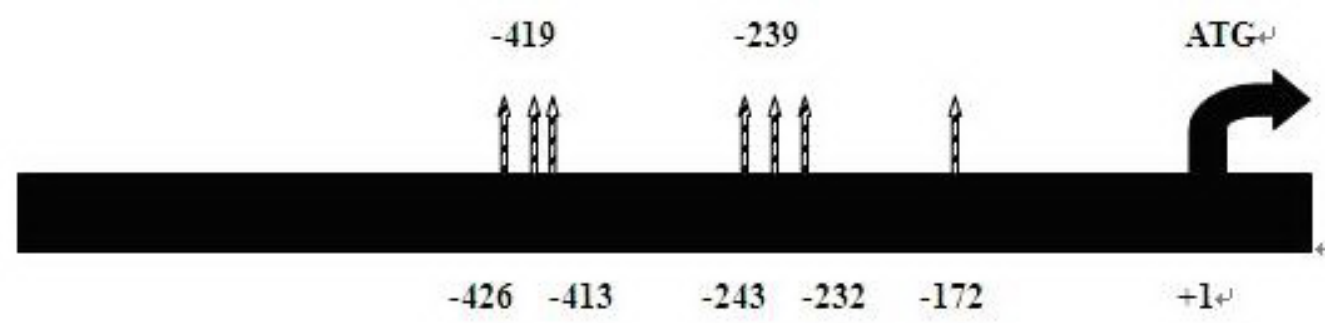

Figure 4. Schematic chart depicting the CpG locations upstream of the HSP70 coding region. The analyzed methylated sites are marked by the number in slash arrow. Positions are numbered with nucleotide $\mathrm{A}$ at the initiator element.

\section{DISCUSSION}

In the present study, the mRNA expression of chicken HSP70 in different tissues under various treatments was analyzed, revealing that $H S P 70$ is heat inducible. The results coincide with those of Gabriel et al. (1996) and Dionello et al. (2001). In addition, mRNA expression of chicken $H S P 70$ was tissue dependent, which agrees with the results of Givisiez et al. (2003). Because no intron is present within HSP70, it can transcribe quickly after heat shock, and it increases sharply with gradual heat stress. Given the results of previous studies of gene polymorphisms that fail to explain clearly the heat-inducible and tissue-dependent nature of HSP70, other mechanisms likely play a role in this phenomenon.

DNA methylation studies in the literature have uncovered profound results (Esteller, 2008; Ushijima and Asada, 2010). Higher methylation levels of the $\mathrm{CpG}$ island within the gene promoter region are known to correlate with gene silencing or downregulation; DNA methylation represses transcription directly by inhibiting the binding of specific transcription factors (Esteller, 2007; Lopez-Serra and Esteller, 2008) and indirectly by recruiting methyl-CpG- 
binding proteins and their associated repressive chromatin remodeling activities (Kuroda et al., 2009). In our study, the methylation level of chicken HSP70 promoter $\mathrm{CpG}$ island was detected using the MassARRAY system, and it revealed hypomethylation of this region in the control, 3-, and 6-h treatment groups in various tissues. The low level of methylation agreed with current data showing that in normal somatic cells, $\mathrm{CpG}$ islands are unmethylated or hypomethylated (Strichman-Almashanu et al., 2002; Song et al., 2005). The results of DNA methylation analysis indicated that regardless of tissue type, the chicken HSP70 promoter showed a similar methylation incident, meaning that the tissue-dependent expression of chicken HSP70 was not associated with the DNA methylation pattern, but interestingly, the methylation level may be negatively associated with mRNA expression because the methylation level of the control and 6-h treatment groups was slightly higher than that of the 3-h treatment group (except in liver tissue), whereas mRNA expression was higher in the 3-h treatment group, and correlation analysis showed that the methylation level was negatively associated with the mRNA level of HSP70 in leg muscle $(\mathrm{P}=0.0124)$. These results indicated that the heat inducibility of $H S P 70$ may be associated with DNA methylation pattern, as it has been demonstrated that heat treatment can change the DNA methylation pattern of certain genes (Yossifoff et al., 2008) However, in the present study, only leg muscle showed a significant negative association between the methylation and mRNA levels, so additional studies are needed to determine the complete relationship between DNA methylation and mRNA expression before and after heat stress.

Methylation levels of $\mathrm{CpG}$ sites within the core promoter were also analyzed. $\mathrm{CpG}$ sites at positions $-426,-419$, and -413 formed a $\mathrm{CpG}$ cluster that was identified as an SP1 transcription site, since none of the differences within tissue groups was significant, but these values were negatively associated with mRNA expression. SP1 is widely expressed in various cell types and plays an important role in the regulation of many housekeeping genes such as those related to nucleic acid metabolism (Safe and Abdelrahim, 2005) and oxidative phosphorylation (Zaid et al., 1999). This transcription factor can also activate gene transcription by preventing the methylation of $\mathrm{CpG}$ islands (Samson and Wong, 2002). In the CpG site at position -172 , which was contained in the HSE, the methylation level was no more than $6 \%$. The HSE is known to play an important role in the transcription of the HSP70. During heat stress, HSFs bind to the HSE to start the transcription of HSP70 and initiate the heat shock response, in which $H S P 70$ is rapidly transcribed and translated. This process may not only be due to the absence of introns in HSP70 but also be associated with the low level of methylation of the HSE, which allows easy access of HSFs to the HSE.

In conclusion, we explored the relationship between promoter $\mathrm{CpG}$ island methylation and HSP70 mRNA expression and demonstrated that the tissue-dependent expression of chicken HSP70 has no relationship with the promoter CpG island methylation pattern. However, the results hinted that DNA methylation may play a role in the heat-inducible nature of $H S P 70$ because of its negative association with mRNA expression of HSP70. Additional studies should be carried out to confirm this phenomenon and indentify the mechanism through which DNA methylation affects the expression of HSP70.

\section{ACKNOWLEDGMENTS}

We thank members of our laboratory for discussions and Dr. David Cushley for comments on the manuscript. Research supported by the National Natural Sciences Foundation of China (\#30972093). 


\section{REFERENCES}

Bird A (2002). DNA methylation patterns and epigenetic memory. Genes Dev. 16: 6-21.

Brena RM, Huang TH and Plass C (2006). Quantitative assessment of DNA methylation: Potential applications for disease diagnosis, classification, and prognosis in clinical settings. J. Mol. Med. 84: 365-377.

Dai Z, Zhu WG, Morrison CD, Brena RM, et al. (2003). A comprehensive search for DNA amplification in lung cancer identifies inhibitors of apoptosis cIAP1 and cIAP2 as candidate oncogenes. Hum. Mol. Genet. 12: 791-801.

Dionello NJL, Ferro JA, Macari M, Rutz F, et al. (2001). Effect of acute heat stress on hepatic and cerebral messenger RNA heat shock protein 70 and heat shock protein 70 level of broiler chicks from 2 to 5 days old of different strains. Rev. Bras. Zootec. 5: 1506-1513.

Ehrich M, Nelson MR, Stanssens P, Zabeau M, et al. (2005). Quantitative high-throughput analysis of DNA methylation patterns by base-specific cleavage and mass spectrometry. Proc. Natl. Acad. Sci. U. S. A. 102: 15785-15790.

Esteller M (2007). Epigenetic gene silencing in cancer: the DNA hypermethylome. Hum. Mol. Genet. 16 Spec No 1: R50-R59.

Esteller M (2008). Epigenetics in evolution and disease. Lancet 372: S90-S96.

Gabriel JE, Ferro JA, Stefani RM, Ferro MI, et al. (1996). Effect of acute heat stress on heat shock protein 70 messenger RNA and on heat shock protein expression in the liver of broilers. Br. Poult. Sci. 37: 443-449.

Givisiez PEN, Furlan RL, Malheiros EB and Macari M (2003). Incubation and rearing temperature effects on Hsp70 levels and heat stress response in broilers. Can. J. Anim. Sci. 2: 213-220.

Guerreiro EN, Giachetto PF, Givisiez PEN, Ferro JA, et al. (2004). Brain and hepatic Hsp70 protein levels in heatacclimated broiler chickens during heat stress. Braz. J. Poult. Sci. 6: 201-206.

Hartl FU (1996). Molecular chaperones in cellular protein folding. Nature 381: 571-579.

Kregel KC (2002). Heat shock proteins: modifying factors in physiological stress responses and acquired thermotolerance. J. Appl. Physiol. 92: 2177-2186.

Kuroda A, Rauch TA, Todorov I, Ku HT, et al. (2009). Insulin gene expression is regulated by DNA methylation. PLoS One 4: e6953.

Lopez-Serra L and Esteller M (2008). Proteins that bind methylated DNA and human cancer: reading the wrong words. Br. J. Cancer 98: 1881-1885.

Maak S, Melesse A, Schmidt R, Schneider F, et al. (2003). Effect of long-term heat exposure on peripheral concentrations of heat shock protein 70 (Hsp70) and hormones in laying hens with different genotypes. Br. Poult. Sci. 44: 133-138.

Mahmoud KZ (2000). Genetic and Environmental Variations of Chicken Heat Shock Proteins. PhD thesis, North Carolina State University, North Carolina.

Mayer MP and Bukau B (2005). Hsp70 chaperones: cellular functions and molecular mechanism. Cell Mol. Life Sci. 62: 670-684.

Mazzi CM, Ferro MIT, Coelho AAD, Savino VJM, et al. (2002). Effect of heat exposure on the thermoregulatory responses of selected naked neck chickens. Arq. Bras. Med. Vet. Zootec. 54: 35-41.

Mazzi CM, Ferro JA, Ferro MIT, Savino VJM, et al. (2003). Polymorphism analysis of the hsp70 stress gene in Broiler chickens (Gallus gallus) of different breeds. Genet. Mol. Biol. 3: 275-281.

Robertson KD and Wolffe AP (2000). DNA methylation in health and disease. Nat. Rev. Genet. 1: 11-19.

Safe S and Abdelrahim M (2005). Sp transcription factor family and its role in cancer. Eur. J. Cancer 41: 2438-2448.

Samson SL and Wong NC (2002). Role of Sp1 in insulin regulation of gene expression. J. Mol. Endocrinol. 29: 265-279.

Song F, Smith JF, Kimura MT, Morrow AD, et al. (2005). Association of tissue-specific differentially methylated regions (TDMs) with differential gene expression. Proc. Natl. Acad. Sci. U. S. A. 102: 3336-3341.

Straussman R, Nejman D, Roberts D, Steinfeld I, et al. (2009). Developmental programming of CpG island methylation profiles in the human genome. Nat. Struct. Mol. Biol. 16: 564-571.

Strichman-Almashanu LZ, Lee RS, Onyango PO, Perlman E, et al. (2002). A genome-wide screen for normally methylated human $\mathrm{CpG}$ islands that can identify novel imprinted genes. Genome Res. 12: 543-554.

Ushijima T and Asada K (2010). Aberrant DNA methylation in contrast with mutations. Cancer Sci. 101: 300-305.

Wang S and Edens FW (1998). Heat conditioning induces heat shock proteins in broiler chickens and turkey poults. Poult. Sci. 77: 1636-1645.

Watanabe Y and Maekawa M (2010). Methylation of DNA in cancer. Adv. Clin. Chem. 52: 145-167.

Xing JY, Kang L, Hu Y, Jiang YL, et al. (2011). Effect of dietary betaine supplementation on mRNA expression and promoter CpG methylation of lipoprotein lipase gene in laying hens. J. Poult. Sci. 3: 224-228.

Xu Q, Zhang Y, Sun D, Wang Y, et al. (2007). Analysis on DNA methylation of various tissues in chicken. Anim. Biotechnol. 18: 231-241. 
Yossifoff M, Kisliouk T and Meiri N (2008). Dynamic changes in DNA methylation during thermal control establishment affect CREB binding to the brain-derived neurotrophic factor promoter. Eur. J. Neurosci. 28: 2267-2277.

Zaid A, Li R, Luciakova K, Barath P, et al. (1999). On the role of the general transcription factor Sp1 in the activation and repression of diverse mammalian oxidative phosphorylation genes. J. Bioenerg. Biomembr. 31: 129-135.

Zhang X, Du H and Li J (2002). Single Nucleotide Polymorphism of Chicken Heat Shock Protein 70 Gene. 7th World Congress on Genetics Applied to Livestock Production, Montpellier.

Zhen FS, Du HL, Xu HP, Luo QB, et al. (2006). Tissue and allelic-specific expression of hsp70 gene in chickens: basal and heat-stress-induced mRNA level quantified with real-time reverse transcriptase polymerase chain reaction. $\mathrm{Br}$. Poult. Sci. 47: 449-455. 